\title{
Bacteriocins of Lactic Acid Bacteria
}

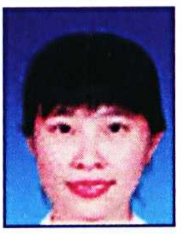

Lactic acid bacteria ( $L A B$ ) have long been used in food preservation through fermentation process. LAB normaliy predominate the microbial flora of many oriental and western food products, which normally use dairy, meat and vegetable as raw materials. Certain $\angle A B$ have the ability to produce bacteriocin-a proteinaceous antimicrobial compound. The $L A B$ and their antimicrobial compounds have been consumed together with fermented food products for centuries without exhibiting any deleterious health effect, therefore LAB have been granted as Generally Regarded As Safe (GRAS) bacteria by FAO and WHO.

Bacteriocin compounds that we discovered from locally isolated $L A B$ are readily hydrolysed by proteolytic enzymes, such as trypsin, $a$ - and b-chymotrypsin, proteinase $K$ and papain. Hence, they are easily hydrolysed in the gastrointestinal tract of consumers as for other protein nutrient without giving any harmful health impact to the consumers. Our studies also demonstrated that the bacteriocins that we obtained from locally isolated LAB have capability to inhibit many species of pathogens (broad inhibitory spectrum characteristics), for instance, Bacillus cereus, Escherichia coli, Streptococcus pneumoniae, Staphylococcus aureus, Salmonella typhimurium, Enterococcus feacalis, Enterococcus faecium and Listeria monocytogenes. Besides that, the bacteriocin compound under studied also could withstand high temperature up to $121^{\circ} \mathrm{C}$ for $15 \mathrm{~min}$ and below $15^{\circ} \mathrm{C}$ for 60 days and they are also tolerable to broad pH range, between $\mathrm{pH}$ 2-5 (acidic $\mathrm{pH})$ and $\mathrm{pH} 7-8($ basic $\mathrm{pH}$ ). Base on the their versatile characteristics, they possess vast potential to be used as natural and consumer friendly biopreservative in various food industries, such as minimally processed and refrigerated foods, heat-treated foods, alkaline and acidic food products.

Most of the Malaysia food products are manufactured by Small Manufacturing Industries or domestic, hence, the quality is usually not consistent and easily perishable. Thus, chemical additives, such as sulphur dioxide, benzoic acid, sorbic acid, nitrate and nitrite are generally used to extend the shelf life of food products. These chemical additives may cause toxicity and have harmful health impact. Many food poisoning incidents due to food additives or pathogens have been reported every year, either locally or worldwide. Therefore there is an increasing interest in using bacteriocins as natural food preservatives worldwide. In addition, the LAB that capable of producing bacteriocin also have vast potential to be a good candidate as well-defined starter culture for local fermented food industry and as probiotic species for human and livestock industries. Beside the application as biopreservatives in food industry, the bacteriocin compounds also have vast potential to be used as feed additives in livestock industries to replace the usage of antibiotics as growth promoter. The use of antibiotics as growth promoter will cause resistance in bacteria, which is not environmentally friendly. Based on the broad inhibitory spectrum of bacteriocins, they also exhibit vast potential in antiseptic cream and cosmetic formulation to solve skin problem. LAB that possesS GRAS status will facilitate the commercialization of the bacteriocin and bacteriocin-producing $\angle A B$. $L A B$ and their metabolites appear to be safe and may not have to undergo the stringent and extensive testing that is generally required for other new compounds before its commercialization. 


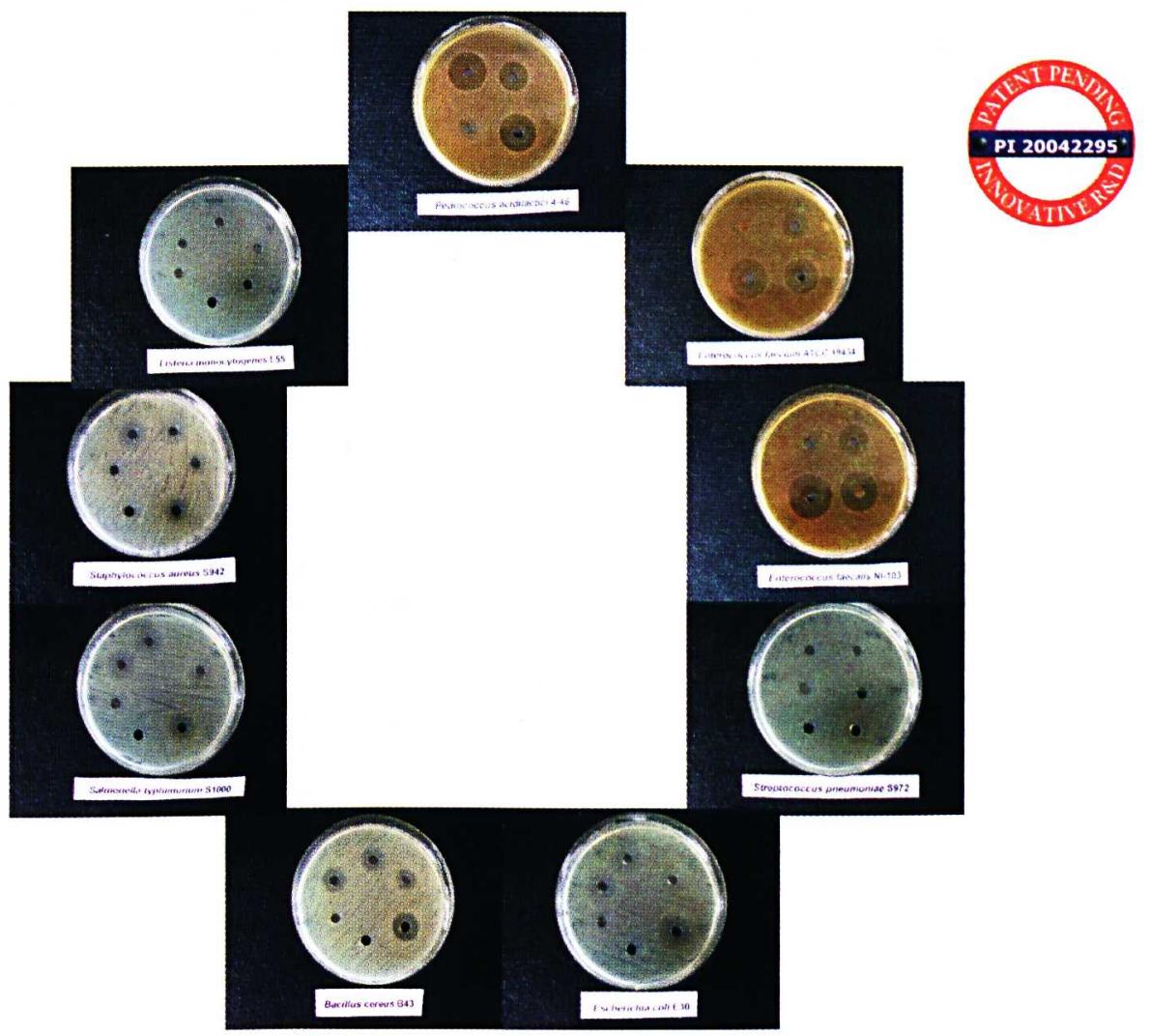

Antagonistic Spectrum of Bacteriocin

For further information, kindly contact:

Assoc. Prof. Dr. Foo Hool Ling

Department of Bioprocess Technology

Faculty of Biotechology and Biomolecular Sciences

Universiti Putra Malaysia

437(30) UPM, Serdang. Sctangor

Malaysia

Tel: $+603894683+3$

Fax: +60389430913

E-mail:hlfoo@fsb.upm.edu.my or hlfoo@putra.upm.edu.my 